\title{
Nanomechanical and Structural Properties of Native Cellulose Under Compressive Stress
}

\author{
Raúl Quesada Cabrera, ${ }^{\mathrm{a}, \mathrm{b}}$, Filip Meersman ${ }^{\mathrm{c},}$, Paul F. McMillan, ${ }^{\mathrm{a},}$, Vladimir Dmitriev ${ }^{\mathrm{b}}$ \\ ${ }^{a}$ Department of Chemistry, University College London, 20 Gordon Street, London WC1H \\ OAJ, United Kingdom, ${ }^{b}$ Swiss-Norwegian Beamlines at ESRF, Boite Postale 220, F-38043 \\ Grenoble, France, 'Department of Chemistry, Katholieke Universiteit Leuven, \\ Celestijnenlaan 200F, B-3001 Leuven, Belgium
}

*Corresponding authors. Email: filip.meersman@chem.kuleuven.be. Tel: +32-16-327355. Fax: +32-16-327990. Email: p.f.mcmillan@ucl.ac.uk. Tel: +44-20-76794610. Fax: +44-2076797463.

\begin{abstract}
Cellulose is an important biopolymer with applications ranging from its use as an additive in pharmaceutical products to the development of novel smart materials. This wide applicability arises in part from its interesting mechanical properties. Here we report on the use of high pressure X-ray diffraction and Raman spectroscopy in a diamond anvil cell to determine the bulk and local elastic moduli of native cellulose. The modulus values obtained are $20 \mathrm{GPa}$ for the bulk modulus, and 200-355 and $15 \mathrm{GPa}$ for the crystalline parts and the overall elastic (Young's) modulus, respectively. These values are consistent with those calculated from tensile measurements. Above $8 \mathrm{GPa}$ the packing of the cellulose chains within the fibres undergoes significant structural distortion, whereas the chains themselves remain largely unaffected by compression.
\end{abstract}




\section{Introduction}

Cellulose is an important structural biopolymer in plants. Because of its abundance this polymer of glucose also represents a major renewable source for the production of biofuel and other chemicals. ${ }^{1,2}$ Cellulose and its derivatives, which include nitrocellulose and methyl cellulose, are used in a wide range of applications in food and pharmaceutical industries. More recently, cellulose has been used in the development of smart materials such as electroactive paper and mesoporous chiral nematic structured silica materials. ${ }^{3-6}$ Amongst the properties that make cellulose such a versatile material are its biocompatibility and high thermal stability, its potential for chemical modification, and high mechanical strength and elastic modulus values. These latter properties make it an ideal component in many nanocomposites. $^{4}$

Cellulose fibres consist of an initimate mixture of crystalline and amorphous regions, at the nanoscale. ${ }^{4,7}$ The overall degree of crystallinity of cotton fibres, for instance, is estimated to be $40-45 \%$ and they seem to contain small crystallites $(4-5 \mathrm{~nm})$ embedded within an amorphous cellulose matrix. ${ }^{8}$ From this perspective, cellulose can be regarded as a nanocomposite structural material and its most interesting mechanical properties can be understood as arising from interactions between the crystalline and amorphous regions, as well as from the properties of these regions themselves. Typical values determined for the elastic or Young's modulus of plant cellulose fibres have been determined to range between 20-30 GPa, but extending up to $138 \mathrm{GPa}$ for highly crystalline cellulose. Such values are generally obtained from tensile measurements. ${ }^{6,9-11}$ Only a very few studies have considered the effects of compressive loading on cellulose, that yields complementary information on the elastic properties. Knowledge of the mechanical behavior under compressive loading is essential for many applications of cellulose, as in structural reinforcing materials, or in drug tablets for which cellulose is used as binding material and that are produced by compression at high pressures. ${ }^{12,13}$ Moreover, it has been shown that at high temperature and moderate pressures (355-400 $\left.{ }^{\circ} \mathrm{C}, 25-33 \mathrm{MPa}\right)$ native cellulose can be dissolved in supercritical water and subsequently precipitated as cellulose II, a polymorph of cellulose I. ${ }^{14}$

Here we report on the structural behavior of native cellulose studied under extreme pressure conditions (up to $16 \mathrm{GPa}$ ) using synchrotron X-ray diffraction and Raman spectroscopy in a diamond anvil cell. ${ }^{15}$, and the data were analyzed to determine its mechanical propertiesOur results may indicate a transformation into a high-pressure form of cellulose above $8 \mathrm{GPa}$. Moreover, the analysis of the X-ray diffraction and Raman data allows us to estimate and evaluate various elastic moduli reported for cellulose I.

The present study is also partly motivated by our recent work on amyloid fibrils, which are protein-based nanofibres, ${ }^{15,16}$ where we employed the same methodology as used in this work to determine their bulk modulus and estimate the Young's modulus. Although the use of such high pressure techniques is a common method for the determination of the elastic moduli in minerals and other solid state materials, it has not been previously applied to the study of large biological macromolecules. In our work, we showed that our data on the mechanical properties of amyloid fibrils were in good agreement with those obtained by atomic force microscopy (AFM) in a tensile regime. Extracting information on the elastic properties from AFM measurements requires a number of assumptions to be made. ${ }^{16,17}$ In particular, the purely tensile stress-strain regime in which the Young's modulus is properly defined for a rod while maintaining constant diameter is only approximately attained, and this must be modelled from the actual data that contain flexional components and shape changes occur. 
Our approach provides direct information on the volume compressibility or bulk modulus obtained within a hydrostatic compressional environment. Because the shear modulus is generally small in these materials, this can be related to the Young's modulus if the compressive and tensile behavior can be thought to mirror each other, in the limit of small strains. In this context cellulose is a good model system to validate both approaches by comparison of the obtained elastic moduli with those from tensile measurements.

\section{Material and Methods}

Sample. Medium-sized cotton cellulose fibres were purchased from Sigma (Bornem, Belgium) and used without further treatment.

Pressure cells. Screw-driven diamond anvil cells were used for the application of pressure. Type IIa diamond anvils were selected with 600 or 300- $\mu \mathrm{m}$ culet diameters for in situ X-ray diffraction and Raman spectroscopy studies, respectively. The sample was contained in stainless steel gaskets that were pre-indented and laser-drilled with hole diameters of 150-400 $\mu \mathrm{m}$. Small ruby chips were added for in situ determination of pressure by the ruby fluorescence method. All experiments were performed at ambient temperature and without pressure transmitting medium.

X-ray diffraction. Synchrotron X-ray diffraction and Raman data were collected at the Swiss-Norwegian Beam Lines (BM01) at the ESRF, France. The X-ray beam $(\lambda=0.7003 \AA)$ was collimated to approximately $100 \times 100 \mu \mathrm{m}^{2}$. A MAR345 area detector was used in the Xray diffraction experiments. The sample-to-detector distance $(351.45 \mathrm{~mm})$ was determined using $\mathrm{LaB}_{6}$ as a calibrant. Calibration and data radial integration to obtain one-dimensional scans of the azimuthal intensity distribution was carried out using Fit2D software. ${ }^{18}$ For the determination of unit cell parameters the data were fitted using the program Fullprof. ${ }^{19}$

Raman spectroscopy. Micro-Raman experiments were performed using a Renishaw ${ }^{\circledR}$ system with CCD detector and He-Ne laser excitation (785 nm, $30 \mu \mathrm{W}$ output power). The spectra were fitted using Origin 7.0 with Gaussian/Lorentzian functions to determine the peak positions accurately.

\section{Results and Discussion}

\section{Pressure induces a distorted phase of cellulose above $8 \mathrm{GPa}$}

A typical two-dimensional X-ray diffraction pattern of cellulose I obtained at ambient conditions is shown in Figure 1, along with the main lattice planes giving rise to the characteristic diffraction rings indicated by the structural model at right. The fact that the diffraction pattern consists of complete rings rather than arcs indicates that the cellulose fibers are not aligned. The corresponding integrated pattern is shown as a plot of intensity vs $2 \theta$ value in Figure 2. The interchain distances are given by the main reflections at 5.93, 5.33 and $3.89 \AA$ that correspond to planes [1-10], [110] and [200], respectively. Other intense reflections at 4.31 and $2.58 \AA$ correspond to planes [012] and [004] within the glucose chains. This is a typical pattern of the cotton-ramie type cellulose, which consists mainly of the $\mathrm{I}_{\beta}$ allomorph. ${ }^{14,20,21}$ Consistent with this the unit cell of native cellulose at ambient conditions was found to be monoclinic (P112 1 ) with lattice parameters $a=7.82 \AA, b=8.26 \AA$, $c=10.40$ $\AA$ and $\gamma=96.3^{\circ}$ (Fig. 2). Note that the cellulose fibre axis is parallel to the $c$ axis, whereas the cellulose chains are hydrogen-bonded into planar sheets along the $b$ axis. These sheets then assemble into stacks along the $a$ axis with van der Waals interactions between the layers. ${ }^{22}$ 

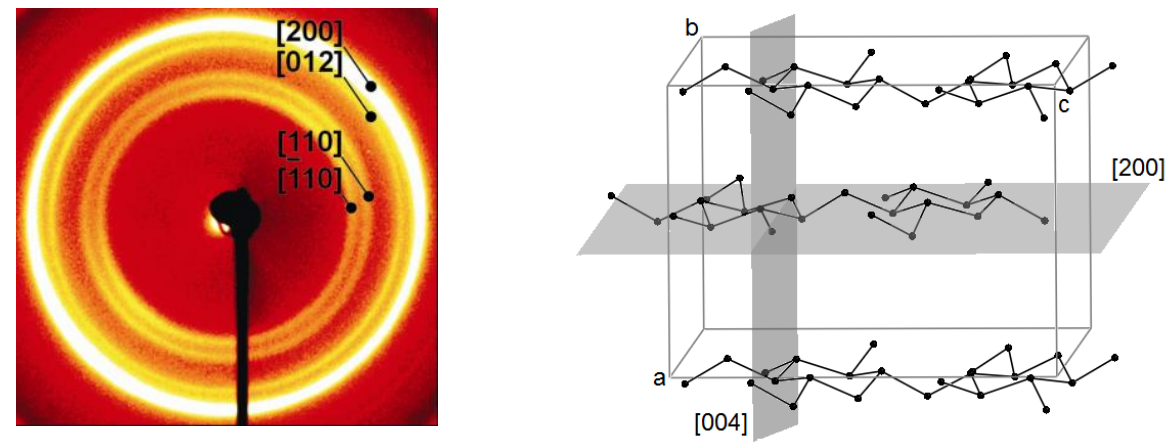

Figure 1. X-ray diffraction pattern of native cellulose at ambient pressure (A) and a schematic representation of the unit cell with the [200] and [004] reflection planes in grey (B). The unit cell was drawn using the program Mercury®.

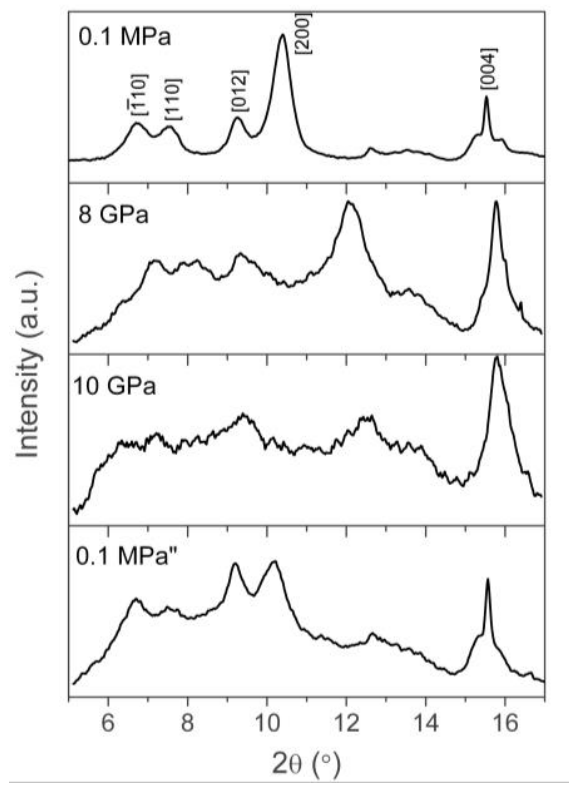

Figure 2. X-ray diffraction patterns of native cellulose at ambient, high-pressure (nonhydrostatic) and recovered conditions ( $0.1 \mathrm{MPa})$.

As the pressure is increased, most reflection peaks become broader and less intense, indicating an increasing structural disorder upon compression, as is also suggested by the changes in background intensity (Fig. 2). The evolution of the main reflections and the corresponding lattice parameters upon compression is shown in Figure 3. There is a significant shift of the [200] reflection, as the distances between the planar sheets are largely affected upon extreme compression. This behavior is typical of sheet-like structures. ${ }^{15,23}$ Interestingly, although broader, the [004] reflection remains relatively sharp and intense even at $10 \mathrm{GPa}$ where most diffraction features have almost disappeared (Fig. 2). This suggests that the cellulose chains remain largely unaffected by the compressive stress. However, there is an increase in the monoclinic angle $\gamma$ with pressure, reaching a plateau above $3 \mathrm{GPa}$ until it displays a sudden increase near $8 \mathrm{GPa}$ (Fig. 3). One possible interpretation of this change in $\gamma$ angle is the occurrence of some shearing distortion within the planar sheets, indicating that the arrangement of the cellulose chains within the fibre becomes highly inhomogeneous as evidenced by the X-ray diffraction patterns at these pressures (Fig. 2). Such a shear distortion could provide a mechanism to dissipate mechanical energy, thereby preventing failure of the 
cellulose fibre. ${ }^{24}$ The long-range changes in the structure are irreversible, as can be observed from the pattern obtained upon recovery at ambient conditions (Fig. 2).
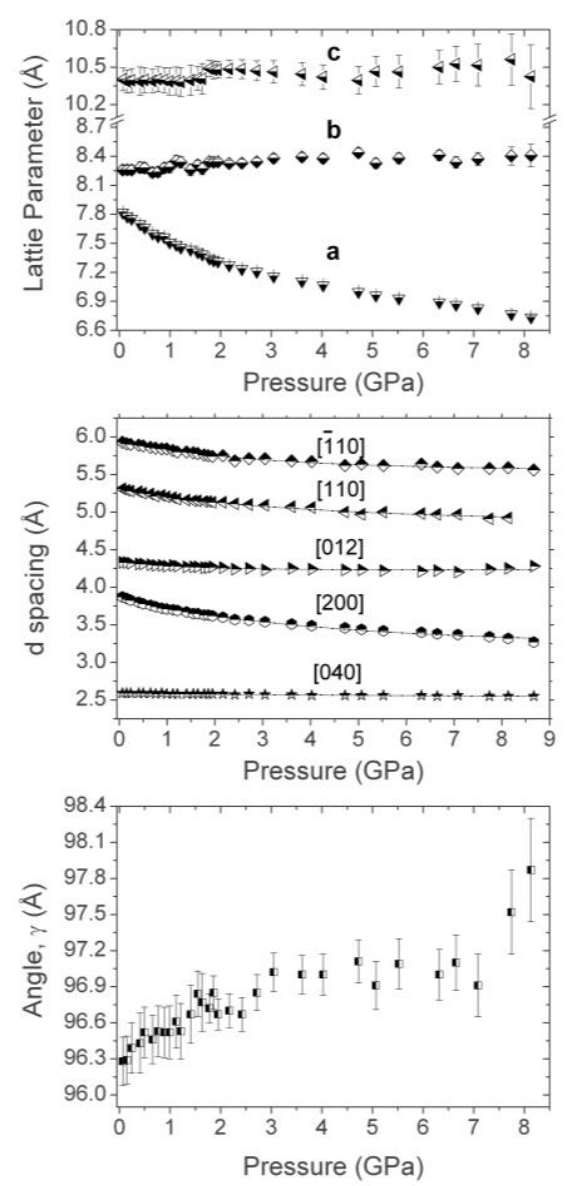

Figure 3. Evolution of the main cellulose I lattice parameters (A), XRD reflections (B) and monoclinic angle $\gamma(\mathrm{C})$ upon compression stress. In (A) any error in the lattice parameter $a$ is less than the size of the symbols.

Further insight in the local structural behavior of cellulose at high pressures was obtained using Raman spectroscopy. Raman spectra upon compression under non-hydrostatic conditions are shown in Figure 4. The assignment of Raman bands is constrained by the large number of atoms in the cellulose structure, the asymmetry in the saccharide unit and the interaction between neighboring residues, which results in a high degree of band overlapping. ${ }^{25}$ In general, the Raman bands above $800 \mathrm{~cm}^{-1}$ are due to skeletal and ring stretching vibrations, together with methylene, methine and hydroxyl vibrations, whereas skeletal and ring bending modes appear below $800 \mathrm{~cm}^{-1}$. All vibrational bands broaden and shift to higher energy upon compression, consistent with previous observations ${ }^{26}$ (Fig. 4). At $8 \mathrm{GPa}$ new phonon bands appear below $200 \mathrm{~cm}^{-1}$ and changes around $1000 \mathrm{~cm}^{-1}$ are detected, becoming more pronounced at $12 \mathrm{GPa}$. There is also a relative intensity decrease and splitting of the bands at 380 and $1095 \mathrm{~cm}^{-1}$. The molecular structure of cellulose can still be recognized up to $16 \mathrm{GPa}$ (Fig. 4), so it is possible that the new vibrational features observed above $8 \mathrm{GPa}$ could indicate the presence of a new cellulose polymorph at high pressures. In addition to the cellulose $\mathrm{I}_{\alpha}$ and $\mathrm{I}_{\beta}$ allomorphs, six other polymorphs have been discovered to date (see, for instance, 25). Cellulose II is obtained by mercerization or regeneration of native cellulose. Reaction of cellulose I and II with liquid ammonia or some amines leads to cellulose III $_{\text {I }}$ and $\mathrm{III}_{\mathrm{II}}$, and heating the latter phases to $206{ }^{\circ} \mathrm{C}$ in glycerol results in cellulose $\mathrm{IV}_{\mathrm{I}}$ and $\mathrm{IV}_{\mathrm{II}}$, 
respectively. No high-pressure polymorphs have been reported to date, although this is now suggested by our new data. However, our X-ray diffraction data do not allow a full structure determination of this high pressure cellulose phase.

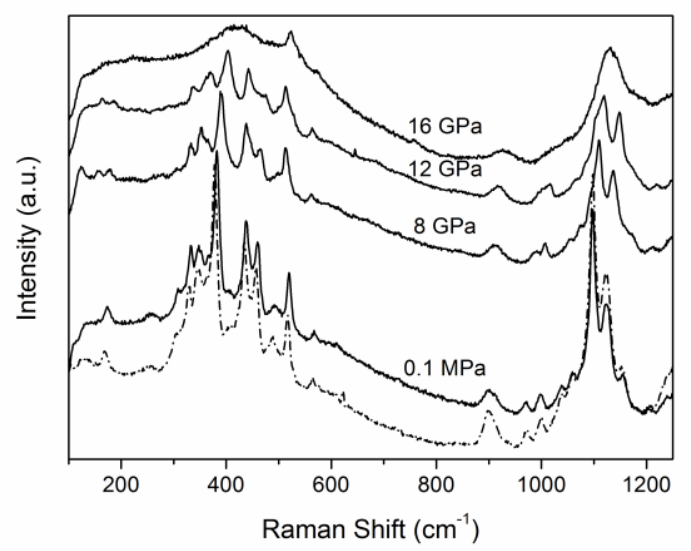

Figure 4. Selected Raman spectra for native cellulose during compression under nonhydrostatic conditions. The bottom (dashed) pattern corresponds to the sample recovered to ambient pressure.

All high pressure transformations observed with Raman spectroscopy are fully reversible, as can be seen from the spectrum of the recovered sample at ambient conditions (Fig. 4). Thus, the irreversible changes observed from our X-ray diffraction results indicate a long-range disordering within the cellulose fibres that gives rise to the broad and weak [200] reflection at a lower $2 \theta$ value than before pressurization. However, the extreme compression treatment of this material has no drastic consequence over the local structure within the cellulose chains upon recovery to ambient conditions.

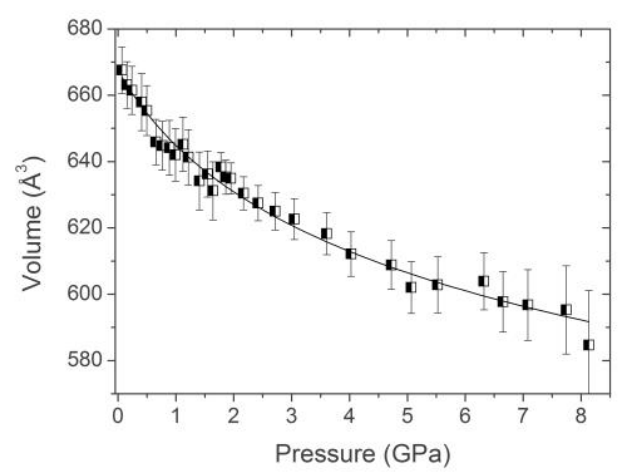

Figure 5. Change in unit cell volume with non-hydrostatic pressure. The solid line represents a fit to the data using the Birch-Murnaghan equation of state.

\section{Cellulose has a bulk modulus of $20 \mathrm{GPa}$}

The theory of elasticity for finite deformation can be used in order to determine the stressstrain behavior of polymer fibres. From the volume-pressure curve (Fig. 5), as obtained by fitting the diffraction reflections and determining the unit cell volume of the overall structure as a function of pressure, it is possible to estimate a bulk modulus for our cellulose sample by using a finite strain Birch-Murnaghan equation of state expanded to the third order (1). ${ }^{28}$ 


$$
P(V)=3 K_{o} f(1+2 f)^{5 / 2}\left(1+\frac{3}{2}\left(K_{o}^{\prime}-4\right) f\right)
$$

The volume strain $f$ is given by

$$
f=\frac{1}{2}\left[\left(\frac{V}{V_{o}}\right)^{-2 / 3}-1\right]
$$

Here $V_{o}$ is the unit cell volume at $0.1 \mathrm{MPa}$ and $V$ represents the corresponding volume at a given pressure $P$. The bulk modulus obtained by fitting to the data is $\mathrm{K}_{0}=19.8( \pm 2.9) \mathrm{GPa}$ and its pressure derivative $\mathrm{K}_{0}{ }^{\prime}=27.8( \pm 6.2)$. The Young's modulus can now be derived by using the following relationship (3):

$$
K=\frac{E}{3(1-2 v)}
$$

Here $E$ is the Young's modulus and $v$ is Poisson's ratio, which is the negative of the ratio of the lateral strain to the axial strain. Using a previously determined value of 0.377 for the Poisson's ratio of cellulose $\mathrm{I}_{\beta},{ }^{29}$ one obtains a Young's modulus of $15.5 \mathrm{GPa}$, which is in good agreement with values for Young's modulus reported in the literature measured under tensile stress conditions (12-27 GPa). ${ }^{4,9,10}$ The values of these moduli depend on the crystallinity of the sample and the interaction of amorphous and crystalline regions, as these determine the change of the unit cell parameters upon compression. A high modulus is expected from highly crystalline cellulose types (e.g. Valonia). The value will also change depending on the relative humidity, which is attributed to a strong vapor adsorption of the amorphous regions in the cellulose structure.

The Young's modulus obtained here and the ones reported in the literature are comparable to the moduli of other biological fibers such as amyloid fibrils and silk, which typically range between 2-40 GPa. ${ }^{30,31}$ There is, however, an interesting difference between these proteinbased fibers based on $\beta$-sheet structures and cellulose. In the latter both the hydrogen bonds and the polymer backbone run along the fibril axis, whereas in amyloid fibrils the backbone runs perpendicular to the fibril axis. Thus in cellulose the 'only' contribution to the mechanical response arises from compression of the intersheet space, whereas in amyloid fibrils, there is a clear contribution from the inter-sheet space, dominated by van der Waals interactions, and the inter-strand interactions, essentially hydrogen bonding. ${ }^{15,16}$

\section{Under compressive stress the crystalline elastic modulus ranges between 200 and $355 \mathrm{GPa}$}

The bulk and Young's moduli reported above should be considered macroscopic moduli that include the contribution of the amorphous regions contained within the cellulose fibres. The $\mathrm{X}$-ray diffraction data, however, can also be analyzed to estimate the elastic modulus of the crystalline regions of cellulose along a single axis. Tensile experiments using aligned fibres take advantage of the shift in reflections such as [004] to obtain the elastic modulus of cellulose crystallites. ${ }^{11}$ Application of the Birch-Murnaghan equation of state (1) to the pressure-induced shift of this reflection (Fig. 3) yields an elastic modulus of $351.5( \pm 52.7)$ $\mathrm{GPa}$. Note that this modulus is defined as either a bulk modulus, if the volume within the crystalline regions is free to relax, or as the compressive equivalent of Young's modulus, if the diameter of each rod-like element is constrained. The results here probably represent a solution that lies partway between these interpretations. For ease of comparison with previous investigations using AFM techniques in the (approximately) tensile regime, we term the resulting elastic modulus determined here as the "Young's modulus".

Raman spectroscopy has been used extensively to study the microdeformation of microcrystalline cellulose fibres. ${ }^{7,32,33}$ This method is based on a two-phase model that regards cellulose as formed by a regular distribution of amorphous and crystalline regions. 
The deformation of cellulose fibres under strain conditions can be studied by the shift of the sharp dominant $1095 \mathrm{~cm}^{-1}$ band in the Raman spectra. ${ }^{9,33,34}$ This band has been assigned to overlapping CCC, COC and $\mathrm{CHO}$ vibrations, including the $\beta-1,4$ glycosidic COC vibration between the glucose moieties, and is taken to be indicative of the deformation of the cellulose backbone structure. The shift of this band can be used for the estimation of the crystalline elastic modulus of cellulose through relation (4) as long as both the relationships between Raman shift and stress $(\mathrm{d}(\Delta v) / \mathrm{d} \sigma)$ and Raman shift and strain $(\mathrm{d}(\Delta v) / \mathrm{d} \varepsilon)$ remain linear. ${ }^{9}$ As illustrated in Fig. 6 this condition is fulfilled up to $8 \mathrm{GPa}$ (Fig. 5).

$$
E=\frac{d \sigma}{d(\Delta v)} \times \frac{d(\Delta v)}{d \varepsilon}=\frac{d \sigma}{d \varepsilon}
$$

It is widely assumed that the applied stress is equal to that developed within the crystalline regions of cellulose. ${ }^{11}$ Similarly to Raman studies of cellulose and polymers carried out under tensile stress, the shift of the $1095 \mathrm{~cm}^{-1}$ band is constant upon compression (Fig. 6). As expected, this shift is much lower in absolute value $\left(1.062 \pm 0.043 \mathrm{~cm}^{-1} / \mathrm{GPa}\right)$ than that reported for tensile experiments $\left(-4.3 \mathrm{~cm}^{-1} / \mathrm{GPa}\right) .{ }^{33} \mathrm{We}$ can obtain the fibre strain for a finite deformation using the expression of Eulerian strain (2).

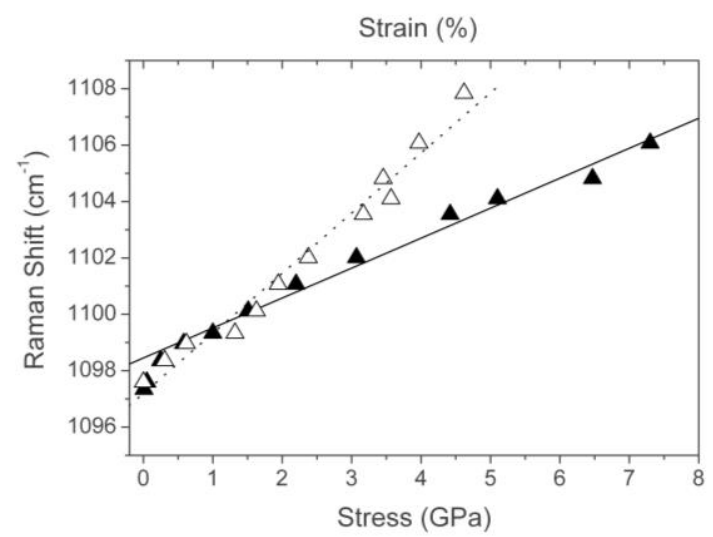

Figure 6. Shift of the Raman band at $1095 \mathrm{~cm}^{-1}$ with stress $(\boldsymbol{\Delta})$ and strain $(\Delta)$ upon compression. The Eulerian strain was extracted from our X-ray diffraction data using equation (4).

The linear relationship between the observed Raman shift and the Eulerian strain measure (Fig. 6) yielded a slope of $2.13 \pm 0.11 \mathrm{~cm}^{-1} / \%$. From these values and equation (3), an elastic modulus value was estimated as $\mathrm{E}=200 \mathrm{GPa}$ for compression up to $8 \mathrm{GPa}$. The result is in good agreement with elastic modulus values for natural cellulose crystals reported in the literature. ${ }^{4,11,35-37}$ From molecular simulations this modulus was calculated to be as high as $171 \mathrm{GPa}$ under compressive deformation. ${ }^{36}$

These high crystalline modulus values reflect the stiffness of the cellulose chain along its long axis and the role of intrachain hydrogen bonds that run parallel to the polymer backbone. Likewise, there is little change in the interchain distances between hydrogen bonded chains as observed from X-ray diffraction. Contrary to tensile experiments the hydrogen bonds are, in first instance, likely to strengthen upon compression. ${ }^{17}$ Hence the modulus values obtained from compression experiments are higher than those from tensile measurements, and should be considered as an upper limit. Compared to the crystalline elastic modulus the bulk and Young's moduli values ( 21 and $15 \mathrm{GPa}$, respectively) are low as they reflect the strong compressibility of the intersheet distances that are devoid of hydrogen bonds. These results highlight the fact that cellulose is a highly mechanically heterogeneous material, because of 
its amorphous and crystalline regions occurring on the nanoscale. The ease of defomation of the amorphous regions results in their contribution to the flexibility and plasticity of cellulose, whereas the highly incompressible crystalline regions contribute to its strength and rigidity. The interaction between the two results in the diverse structural applications of this unique material that have evolved naturally, and are exploited technologically.

\section{Conclusions}

Cellulose is shown to undergo significant structural distortion at high pressures, possibly leading to the adoption of a new phase above $8 \mathrm{GPa}$. The changes observed by $\mathrm{X}$-ray diffraction are not fully reversible, suggesting that the packing of the cellulose chains in the fibers remains distorted after decompression. At the molecular level, however, Raman spectroscopy indicates that the chemical bonds experience the same environment as prior to compression. In addition, we could determine the bulk and elastic moduli of cellulose and found that the obtained values are in excellent agreement with those reported on the basis of tensile experiments. This is an important finding as it indicates that our method is useful for the determination of the mechanical properties of other biological systems and validates our previous findings for amyloid fibrils. ${ }^{15,16}$ It also suggests that compressive and tensile stress mirror each other, as previously reported for amyloid fibrils, ${ }^{17}$ even though under the hydrostatic or non-hydrostatic conditions in the diamond anvil cell the compressive stress is not applied along a single structural axis.

Acknowledgements. F.M. is a postdoctoral research fellow of the Research Foundation Flanders (FWO-Vlaanderen). R.Q.C. was supported by EPSRC Senior Research Fellowship EP/D07357X to P.F.M. The authors acknowledge SNBL for beam time allocation and the SNBL staff for the support.

\section{References and Notes}

(1.) Binder, J.B.; Raines, R.T. Proc. Natl. Acad. Sci. USA 2010, 107, 4516-4521.

(2.) Shen, T.; Gnanakaran, S. Biophys. J. 2009, 96, 3032-3040.

(3.) Shopsowitz, K.E.; Qi, H.; Hamad, W.Y.; MacLachlan, M.J., Nature 2010, 468, 422425.

(4.) Eichhorn, S.J.; Dufresne, A.; Aranguren, M.; Marcovich, N.E.; Capadona, J.R.; Rowan, S.J.; Weder, C.; Thielemans, W.; Roman, M.; Renneckar, S.; Gindl, W.; Veigel, S.; Keckes, J.; Yano, H.; Abe, K.; Nogi, M.; Nakagaito, A.N.; Mangalam, A.; Simonsen, J.; Benight, A.S.; Bismarck, A.; Berglund, L.A.; Peijs, T. J. Mat. Sci. 2010, 45, 1-33.

(5.) Kim, J.; Yun, S.; Ounaies, Z. Macromolecules 2006, 39, 4202-4206.

(6.) Sehaqui, H.; Liu, A.; Zhou, Q.; Berglund, L.A. Biomacromolecules 2010, 11, 21952198.

(7.) Kong, K.; Wilding, M.A.; Ibbett, R.N.; Eichhorn, S.J. Faraday Discuss. 2008, 139, 283-298.

(8.) Morosoff, N. J. Appl. Polymer Sci. 1974, 18, 1837-1854.

(9.) Eichhorn, S.J.; Young, R.J. Cellulose 2001, 8, 197-207.

(10.) Kölln, K.; Grotkopp, I.; Burghammer, M.; Roth, S.V.; Funari, S.S.; Dommach, M.; Müller, M. J. Synchrotron Rad. 2005, 12, 739-744.

(11.) Nishino, T.; Takano, K.; Nakamae, K. J. Polymer Sci. B: Polymer Phys. 1995, 33, 1647-1651.

(12.) Suzuki, T.; Nakagami, H. Eur. J. Pharmaceut. Biopharmaceutics 1999, 47, 225-230.

(13.) Fabbiani, F.P.A.; Pulham, C.R. Chem. Soc. Rev. 2006, 35, 932-942. 
(14.) Sasaki, M.; Adschiri, T.; Arai, K. J. Agric. Food Chem. 2003, 51, 5376-5381

(15.) Meersman, F.; Quesada Cabrera, R.; McMillan, P.F.; Dmitriev, V. Biophys. J. 2011, 100, 193-197.

(16.) Meersman, F.; Quesada Cabrera, R.; McMillan, P.F.; Dmitriev, V. High Pressure Res. 2009, 29, 665-670.

(17.) Paparcone, R.; Keten, S.; Buehler, M.J. J. Biomechanics 2010, 43, 1196-1201.

(18.) Hammersley, A.P.; Svensson, S.O.; Hanfland, M.; Fitch, A.N.; Häusermann, D. High Pressure Res. 1996, 14, 235-248.

(19.) Rodríguez-Carvajal, J. Physica B 1993, 192, 55-69.

(20.) Woodcock, C.; Sarko, A. Macromolecules 1980, 13, 1183-1187.

(21.) Wada, M.; Okano, T.; Sugiyama, J. Cellulose 1997, 4, 221-232.

(22.) Nishiyama, Y.; Langan, P.; Chanzy, H. J. Am. Chem. Soc. 2002, 124, 9074-9082.

(23.) Hanfland, M.; Beister, H.; Syassen, K. Phys. Rev. B 1989, 39, 12598-12603.

(24.) Buehler, M.J.; Yung, Y.C. Nat. Mater. 2009, 8, 175-188.

(25.) Gardner, K.H.; Blackwell, J. Biopolymers 1974, 13, 1975-2001.

(26.) Rusli, R.; Eichhorn, S.J. Appl. Phys. Lett. 2008, 93, 033111.

(27.) O'Sullivan, A.C. Cellulose 1997, 4, 173-207.

(28.) Soignard, E.; Shebanova, O.; McMillan, P.F. Phys. Rev. B 2007, 75, 014104.

(29.) Nakamura, K.; Wada, M.; Kuga, S.; Okano, T. J. Polymer Sci. B Polymer Phys. 2004, 42, 1206-1211.

(30.) Xu, Z.; Buehler, M.J. Phys. Rev. E 2010, 81, 061910.

(31.) Knowles, T.P.; Fitzpatrick, A.W.; Meehan, S.; Mott, H.R.; Vendruscolo, M.; Dobson, C.M.; Welland, M.E. Science 2007, 318, 1900-1903.

(32.) Atalla, R.H. Appl. Polym. Symp. 1976, 28, 659-669.

(33.) Eichhorn, S.J.; Sirichaisit, J.; Young, R.J. J. Mat. Sci. 2001, 36, 3129-3135.

(34.) Eichhorn, S.J., Young, R.J.; Yeh, W.-Y. Textile Res. J. 2001, 71, 121-129.

(35.) Tanaka, F.; Iwata, T. Cellulose 2006, 13, 509-517.

(36.) Matsuo, M.; Sawatari, C.; Iwai, Y.; Ozaki, F. Macromolecules 1990, 23, 3266-3275.

(37.) Sakurada, I.; Nukushina, Y.; Ito, T. J. Polym. Sci. 1962, 57, 651-660. 


\section{For Table of contents use only}

Nanomechanical and Structural Properties of Native Cellulose Under Compressive Stress Raúl Quesada Cabrera, Filip Meersman, Paul F. McMillan, Vladimir Dmitriev
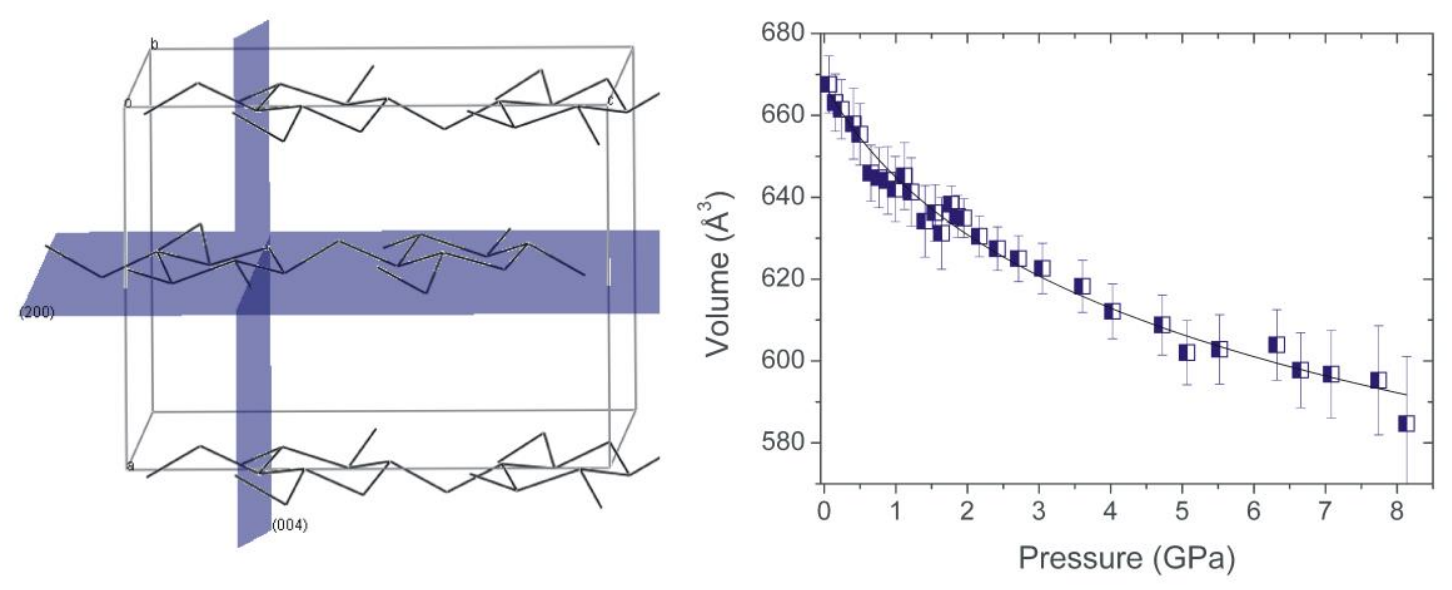